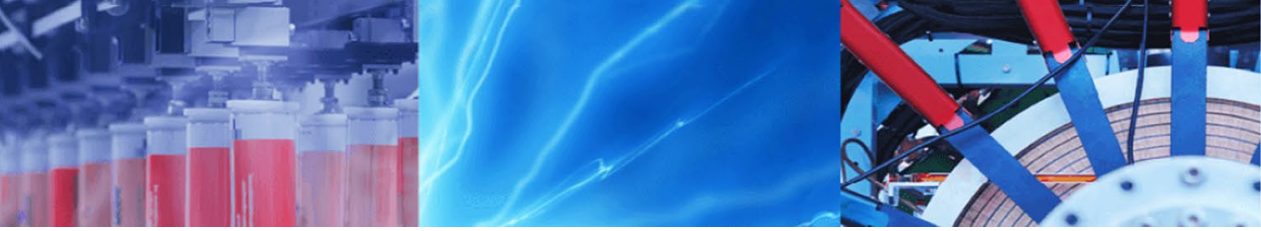

Research Article

\title{
Multi-objective multi-load tandem autonomous guided vehicle for robust workload balance and material handling optimization
}

\author{
Behnam Rahimikelarijani $^{1}\left[\right.$ [ Hamed Fazlollahtabar ${ }^{2} \cdot$ Sina Nayeri $^{3}$
}

Received: 28 August 2019 / Accepted: 4 June 2020 / Published online: 11 June 2020

(c) Springer Nature Switzerland AG 2020

\begin{abstract}
Effective transportation methods in automated manufacturing systems increase efficiency and decrease final product cost. Flexibility of autonomous guided vehicles (AGVs) in performing transportation tasks makes them an important option in automated manufacturing system design. Optimal decisions for configuring tandem AGV system in a real-time mechanism are important. In this study, we propose a nonlinear binary mathematical model to configure a tandem AGV system, where multiple-load AGVs are considered. A multi-objective model has been developed to minimize inter- and intra-loop transportations and to balance workload in different cells based on AGVs performance. Since AGVs performance may change by considering different loading and unloading policies, robust optimization approach has been applied in modeling. Then, a hierarchical methodology has been proposed to solve the optimization problem. Finally, an illustrative example is developed to check the validity of the proposed mathematical model. Sensitivity analyses emphasize the contribution of the work and imply managerial implications for decision makers and policy developers.
\end{abstract}

Keywords Multiple-load AGV · Tandem · Multiple objective · Machine-to-loop assignment · Robust optimization

\section{Introduction}

Material flow made up around $30 \%$ of the total operating expenses within manufacturing system. An effective facility layout and material flow network design can reduce material flow costs about 20\% [1]. Flexibility of automated guided vehicles (AGVs) in design and routing made it as a choice in material handling design.

An AGV was introduced in 1955 for horizontal transportation of goods and material [2], and initially, AGVs were mostly used in manufacturing systems [3], but their application has increased rapidly in production, distribution, warehouses and shipping environment [4]. AGV system can be categorized based on their follow path design [4] into three categories: single loop, tandem configurations and segmented flow configuration. Tandem configuration made up of multiple non-overlapping loops, where in each loop an AGV performing transportations, and interloop transportations will be done with an express loop or a network of conveyers. This design strategy results in less complicated control system, omits collisions and deadlocks and also makes system extension and adding loop easier. Tandem configuration was proposed by [5] as presented in Fig. 1.

The main problems in tandem AGV designs are categorized as follows [6];

- Determining number of loops (fleet size)

- Assigning workstations to loops

- Arranging stations in each loop

- Specifying optimum transfer point between loops

- Flow direction in loops (clockwise or counterclockwise)

\footnotetext{
$\triangle$ Behnam Rahimikelarijani, brahimikelar@lamar.edu; Hamed Fazlollahtabar, hfazl@du.ac.ir; Sina Nayeri, sinany1992@gmail.com| ${ }^{1}$ Department of Industrial Engineering, School of Engineering, Lamar University, Beaumont, TX, USA. ${ }^{2}$ Department of Industrial Engineering, School of Engineering, Damghan University, Damghan, Iran. ${ }^{3}$ Department of Industrial Engineering, Babol Noshirvani University of Technology, Babol, Iran.
} 


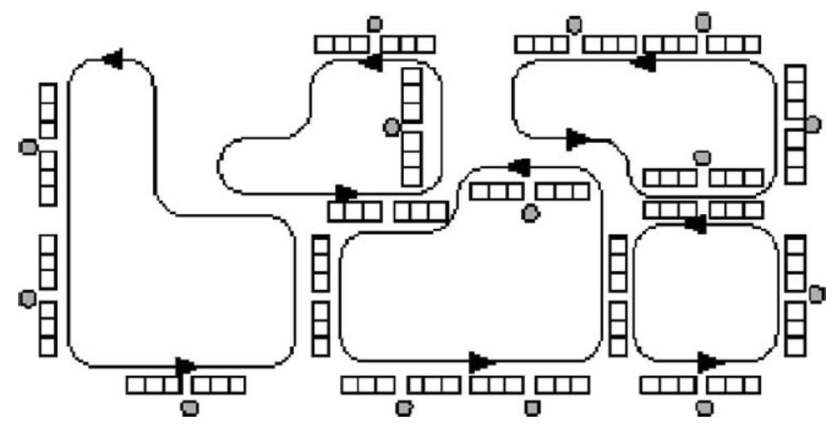

Fig. 1 Tandem configuration as proposed by [5]

Assigning stations in the system into a set of zones is a key problem in the design of tandem systems [7], as mentioned before efficient design of material handling system, and facility layout can reduce manufacturing cost around $20 \%$, which is the main focus of this study.

Different models have been proposed to optimized design of tandem layout system, mainly studies considering balancing workload between stations and minimizing inter- and intra-loop transportation as their main objective functions. Minimizing inter-loop work flow and using balancing strategies were considered in [8-10]. Minimizing maximum workload is another strategy which considered by $[7,11]$. Minimizing both loaded and empty flow between stations is another approach that studied in [1]. Considering total waiting time of all workstations, which is equivalent to minimizing the total lateness of each zone in their objective function, is proposed by [12].

After selecting AGV in material handling system, decisions should be made on AGV capacity. One of the disadvantages of the tandem configuration is loss of efficiency due to transfer stations [13], which could overcome by using multiple-load AGVs. Increasing capacity of AGV is mentioned in many studies. In [14], it is indicated that increasing AGV capacity from one to two leads to fleet reduction. In [15], it is shown that an increase in AGV load results in decreasing mean output time. However, this decrease in mean output time is nonlinear. Researchers in [13] used simulation model to show that the AGV performance will increase as the number of loads increase, but this increase is nonlinear, and they showed that the performance index for one-load AGV is one, but for two-load AGV is 1.91 and for three-load AGV is 2.74. As [16] mentioned, most of the researches considered single-load AGV and few researches consider multiple-load AGV systems [9, 13, 17-19], which is one of the main focuses of this study.

One of the main problems in designing an AGV system is that the performance of multiple-load tandem AGV system (MLTAGVS) will change under different work strategies [13] (first come first serve (FCFS), last come first serve (LCFS) and random serve). This issue in designing multiple-load AGV is not frequently considered in past researches. The main contribution of this study is threefold; first, a new model is proposed that comprises three decisions in designing a tandem AGV configuration which are minimizing inter-loops, intraloops and AGV capacity cost. Second, AGVs performance in each cell will consider as balancing measure, considering AGV's performance coefficient rather than capacity making the model more real and applicable. Third, by applying robust optimization approach, we assure a robust layout design for different working policies.

The rest of this paper is arranged as follows. In Sect. 2, a detailed problem definition is presented and justified. The robust optimization approach is discussed in Sect. 3 and adapted illustratively for the mathematical formulation. In Sect. 4, a solution methodology is introduced to solve the multi-objective optimization problem and the model is worked out in an example and sensitivity analysis of different parameters is presented. We conclude in Sect. 6.

\section{Problem definition and formulation}

Finding best layout configuration will reduce transportation cost, save energy, reduce transportation system lifetime and reduce final product price. We want to assign a set of workstations and AGVs into cells to minimize transportation cost, inter- and intra-loop transportations and be efficient in confronting different work strategies (Fig. 1). In general, designing an AGV system is like cellformation problems, where we try to minimize inter-cell transportations to reduce perturbations and transportation costs. This problem is derived from cell-formation concepts, in which reducing intra-loops transportation is one of the main goals. In this section, an exact definition of the problem formulation and notation is presented; then, a deterministic nonlinear mathematical model is developed, and afterward, a robust optimization approach is employed to cope with uncertainties. The sets/indices and parameters used in the MLTAGVS model are as follows:

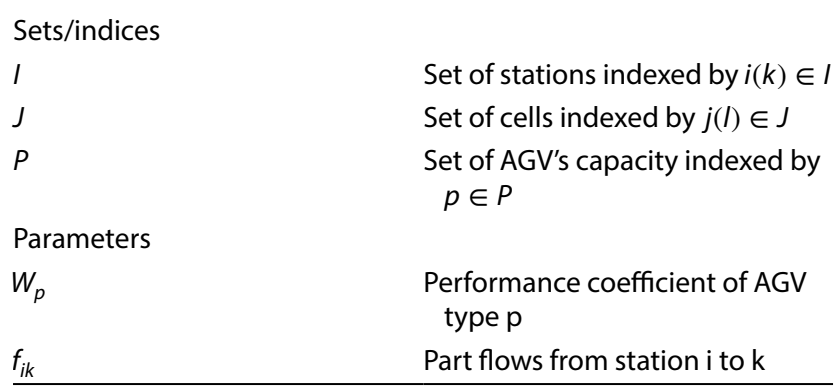




\begin{tabular}{ll}
\hline$f_{i}$ & $\begin{array}{l}\text { Total flow to station } i\left(\sum_{k} f_{i k}\right) \\
\text { Total available time during work } \\
\text { period }\end{array}$ \\
$t_{i}$ & $\begin{array}{c}\text { Mean loading, unloading and } \\
\text { processing time on station } \mathrm{i}\end{array}$ \\
$N$ & $\begin{array}{l}\text { Number of cell } \\
\text { Penalty cost of using AGV type } \mathrm{p}\end{array}$ \\
$\lambda_{p}$ & Number of stations \\
$n$ & Decision variables \\
$X_{i j}$ & $\begin{cases}1 ; & \text { if station } i \text { assigns to cell } j \\
0 ; & \text { otherwise }\end{cases}$ \\
$y_{j p}$ & $\begin{cases}1 ; & \text { if AGV type } p \text { assigns to cell } j \\
0 ; & \text { otherwise }\end{cases}$ \\
$t_{j}^{\prime}$ & Bottleneck station time in cell $j$ \\
\hline
\end{tabular}

Also, the following assumptions are considered for development of the mathematical model.

1. AGVs are bidirectional.

2. There is only one AGV in each cell.

3. There are at least two stations in each loop.

4. Each station can only be assigned to one loop.

5. The number of loops is considered as an input to the algorithm.

6. Only loaded transportations are considered.

7. AGV's performance is considered to make the results more applicable and real.

The problem aim is to form a MLTAGVS which is to minimize inter-loops and intra-loops transportation cost and minimize AGV extending capacity cost and also balancing work load in different cell considering AGV's performance coefficient and available time, so the three elements of MLTAGVS objective function are as follows:

1. Inter-loop transportation costs are considered in (1a). Inter-loop transportation takes place when a part needs to process in more than one cell. This kind of transportation is done with a network of conveyers, so the capacity is not considered in this part. In cellular manufacturing problems, it is usual to minimize interloop transportations to reduce perturbation.

2. Intra-loop transportation costs are presented in (1b). Intra-loop transportation happens when a part needs to process in more than one station in a cell. This part of objective function minimizes inter-loop transportation and at the same time tries to balance work load in cells by considering AGV's load capacity. By minimizing maximum workload, we try to balance the workload in different cells by considering the assigned AGV capacity.
3. Increasing an AGV capacity is considered in (1c). This part limited unnecessary increase in an AGV's capacity by considering penalty cost.

$$
z_{1}=\min \sum_{i=1}^{n} \sum_{j=1}^{N} \sum_{\substack{k=1 \\ i \neq k, j \neq l}}^{n} \sum_{\substack{l=1 \\ i k}}^{N} x_{i j} x_{k l}
$$

$$
z_{2}=\min \left(\max \left(\frac{\sum_{i=1}^{n} \sum_{k=1}^{n} f_{i k} x_{i j} x_{k l}}{\sum_{\substack{p=1 \\ i \neq k, j=l}}^{m} y_{j p} w_{p}}\right)\right)
$$

$z_{3}=\min \sum_{j=1}^{N}\left(\sum_{p=1}^{m} y_{j p} w_{p} \lambda_{p}\right)$

st:

$$
\sum_{j=1}^{N} x_{i j}=1 \quad \text { for } i=1,2, \ldots, n
$$

$\sum_{p=1}^{m} y_{j p}=1 \quad$ for $j=1,2, \ldots, N$

$$
\sum_{i=1}^{n} x_{i j} \geq 2 \text { for } j=1,2, \ldots, N
$$

$t_{j}^{\prime}=\operatorname{Max}_{i}\left\{f_{i} t_{i} x_{i j}\right\} \quad$ for $j=1,2, \ldots, N$

$\sum_{i=1}^{n} x_{i j} \leq \frac{T\left(\sum_{p=1}^{m} y_{j p} w_{p}\right)}{t_{j}^{\prime}}$ for $j=1,2, \ldots, N$

$x_{i j} \in\{0,1\}, \forall i, j$

$y_{j k} \in\{0,1\}, \forall j, k$

Equations (2)-(8) are considered in modeling. Equation (2) ensures that each station will assign to only one cell. Equation (3) guarantees that only one AGV with specific capacity assigns to a cell. Constraint (4) states each cell constructed with more than two stations, Eq. (5) finds the bottleneck station in each cell and applies it in Constraint (6) as a cycle time to check and ensure the ability of a multiple-load AGVs to perform transportation services in planning period, and Eqs. (7) and (8) define types of decision variables. 


\subsection{Linearization}

In this section, linearization techniques are applied to the nonlinear equations of the mathematical model. Multiplication of assigning decision variable $x_{i j} x_{k l}$ in part (1a) and (1b) of objective function needs to be linearized. By using general method in quadratic assignment problem, firstly we define a new variable $\vartheta_{i j k l}=x_{i j} x_{k l}$. Adding this variable requires some additional constraints as follows:

$x_{i j}+x_{j k}-2 \vartheta_{i j k l} \geq 0 \quad \forall i, j, k, l$

$\vartheta_{i j k l} \geq 0 \quad \forall i, j, k, l$

In next step, the minmax objective function in part (1b) is linearized by defining new variable $u$. We need to consider additional constraints to linearize this objective function as follows:

$\min \max f(x)$

s.t : $\quad A x \leq b, x \geq 0$

model (a)

Linearize to

$\min u$
s.t $: A x \leq b, x \geq 0, f(x) \leq u$

After substituting new variables and adding new constraints, the proposed model will be as follows:

$$
\begin{aligned}
& z_{1}=\min \sum_{i=1}^{n} \sum_{j=1}^{N} \sum_{\substack{k=1 \\
i \neq k, j \neq l}}^{n} \sum_{\substack{l=1 \\
i}}^{N} \vartheta_{i j k l} \\
& z_{2}=\min u \\
& z_{3}=\min \sum_{j=1}^{N}\left(\sum_{p=1}^{m} y_{j p} w_{p} \lambda_{p}\right) \\
& \text { st : } \\
& \sum_{j=1}^{N} x_{i j}=1 \text { for } i=1,2, \ldots, n \\
& \sum_{p=1}^{m} y_{j p}=1 \quad \text { for } j=1,2, \ldots N \\
& \sum_{i=1}^{n} x_{i j} \geq 2 \text { for } j=1,2, \ldots, N
\end{aligned}
$$

$$
t_{j}^{\prime}=\operatorname{Max}_{i}\left\{f_{i} t_{i} x_{i j}\right\} \quad \text { for } j=1,2, \ldots, N
$$

$$
\sum_{i=1}^{n} x_{i j} \leq \frac{T \sum_{p=1}^{m} y_{j p} w_{p}}{t_{j}^{\prime}} \text { for } j=1,2, \ldots, N
$$

$x_{i j} \in\{0,1\}$ for $i, j$

$y_{j k} \in\{0,1\}$ for $j, k$

$x_{i j}+x_{j k}-2 \vartheta_{i j k l} \geq 0$ for $i, j, k, l$

$y_{i j k l} \geq 0 \quad$ for $i, j, k, l$

$$
\frac{\sum_{i=1}^{n} \sum f_{i k} x_{i j} x_{k l}}{\sum_{\substack{p=1 \\ i \neq k, j=l}}^{m} y_{j p} w_{p}} \leq u \text { for } j
$$

\section{Robust optimization approach}

Robust optimization is an important subfield of optimization, which protects decision makers against parameter ambiguity and uncertainty [20]. Here, we consider approach introduced by [21] for optimizing problems under uncertainty. Consider the following linear mathematical programming model:

$\min \sum_{i, j} c_{i j} x_{j} \quad \operatorname{Model}(\mathrm{c})$

s.t : $\sum_{j} \tilde{a}_{i j} x_{j} \geq b_{i} \quad \forall i$

$x_{j} \geq 0 \quad \forall j$

where $\tilde{a}_{i j}$ are the uncertain coefficients. Consider $J_{i}$ as a set of uncertain coefficients of the ith constraint; then, each uncertain parameter $\tilde{a}_{i j}, j \in j_{i}$ is a symmetric and bounded value in interval $\left[a_{i j}-\hat{a}_{i j}, a_{i j}+\hat{a}_{i j}\right]$, where $a_{i j}$ is the nominal value and $\hat{a}_{i j}$ denotes the perturbation in uncertain parameter $\tilde{a}_{i j}[22]$. For each constraint $i$, a parameter $\Gamma_{i}$ is introduced to adjust uncertainty level in each constraint by varying in interval of $\left[0,\left|J_{i}\right|\right]$, and this parameter is called uncertainty budget and controls the degree of conservatism and is not necessarily integer. The robust formulation objects are to protect in cases, where up to $\left|\Gamma_{i}\right|$ parameters are allowed to change and coefficient $\tilde{a}_{i j}$ changes by $\left(\Gamma_{i}-\left[\Gamma_{i}\right]\right) \hat{a}_{i j}$ [22]. When $\left|\Gamma_{i}\right|$ is equal to zero, the constraints are equal to nominal problem. Similarly when $\left|\Gamma_{i}\right|$ is equal to $\left|J_{i}\right|$, the robust model acts as conservative 
as in the robust formulation of [23]. The nonlinear form of uncertain model (c) can be written as follows [21]:

$\min \sum_{i, j} c^{\prime} x_{j} \quad \operatorname{Model}(\mathrm{d})$

s.t $: \sum_{j} a_{i j} x_{j}-\max _{\Omega}\left\{\sum_{j \in s_{i}} \hat{a}_{i j}+\left(\Gamma_{i}-\left[\Gamma_{i}\right]\right) \hat{a}_{i t_{i}} x_{j}\right\} \geq b_{i} \quad \forall i$

$x_{j} \geq 0 \quad \forall j$

In model (d), $\Omega=\left\{S_{i} \cup t_{i}\left|S_{i} \subseteq j_{i},\right| S_{i} \mid=\left[\Gamma_{i}\right], t_{i} \in j_{i} \backslash S_{i}\right\}$ defines the uncertainty set. $S_{i}$ determines coefficients changed by $\hat{a}_{i j}$, and $t_{i}$ indicates coefficients may change by $\left(\Gamma_{i}-\left[\Gamma_{i}\right]\right) \hat{a}_{i j}$. Consider $x^{*}$ as the optimal solution of model(d), and [21] proved that the ith constraint is protected by $\beta_{i}\left(x^{*}, \Gamma_{i}\right)=\max _{\Omega}\left\{\sum_{j \in j_{i}} \hat{a}_{i j} x_{i j}^{*} \omega_{i j}\right\}$ against uncertainty. The protection function, $\beta_{i}\left(x^{*}, \Gamma_{i}\right)$, can be written as a linear optimization model as follows:

$\beta_{i}\left(x^{*}, \Gamma_{i}\right)=\max \left\{\sum_{j \in j_{i}} \hat{a}_{i j} x_{i j}^{*} \omega_{i j}\right\} \quad \operatorname{model}(\mathrm{e})$

$\sum_{j \in j_{i}} \omega_{i j} \leq \Gamma_{i}$

$0 \leq \omega_{i j} \leq 1 \quad \forall J \in j_{i}$

Since model (e) is feasible and bounded for all of $\Gamma_{i} \in\left[0,\left|J_{i}\right|\right]$, according to strong duality property, its dual form is feasible and bounded [22]. Replacing the dual form of model (e) in model (d), the robust counterpart of the uncertain linear programming mode $(f)$ is as follows:

$$
\begin{aligned}
& \min \sum_{j} c_{j} x_{j} \quad \operatorname{model}(\mathrm{f}) \\
& \text { s.t }: \sum_{j} a_{i j} x_{j}-\lambda_{i} \Gamma_{i}-\sum_{j \in j_{i}} \mu_{i j} \geq b_{i}, \quad \forall i \\
& \lambda_{i}+\mu_{i j} \geq \hat{a}_{i j}, \quad \forall i, j \in j_{i} \\
& \mu_{i j} \geq 0, \quad \forall i, j \in j_{i} \\
& \lambda_{i} \geq 0 \quad \forall i \\
& x_{i j} \geq 0, \quad \forall i
\end{aligned}
$$

where $\lambda_{i}$ and $\mu_{i j}$ are the dual auxiliary variables.

\subsection{Robust formulation of MLTAGVS problem}

In this section, we use robust optimization approach in combination with proposed mathematical model for MLTAGVS to consider uncertain performance coefficients in modeling. Two are three uncertain parameters, which should take into account; first parameter is $\tilde{w}_{p}$ which can be varied according to loading and unloading policy and work environment. Using simulation [13] showed that an AGV performance will change by considering different capacity, and they calculate AGVs capacity using different loading and unloading policies for one- to threeload AGV. Based on these simulation, the performances are as follows: $w_{1}=1 \tilde{w}_{2}=[1.91-0.06,1.91+0.06]$ and, $\tilde{w}_{3}=[2.74-0.17,2.74+0.17]$ in sequence. Parameter $\tilde{w}_{p}$ existed in part (1c) of objective function and Constraints (6) and (11). The main concern in robust formulation is considering worst case, so part (1c) of objective function customizes as follows:

$Z_{3}=\min \sum_{j=1}^{N}\left(\left(\sum_{p=1}^{m} y_{j p} w_{p}\right)+\max _{\Omega^{p}}\left(\sum_{p \in S^{p}} \hat{w}_{p} y_{j p}\right)\right) \lambda_{p}$

$\Omega^{p}=\left\{S^{p}\left|S^{p} \subseteq j^{p},\right| S^{p} \mid \leq \Gamma^{p}\right\}, j^{p}=\left\{p\left|\hat{w}_{p}\right\rangle 0\right\}, j^{p}=\left\{p\left|\hat{w}_{p}\right\rangle 0\right\}$

The nonlinear function (12) can be written as a protection function discussed in Sect. 3 and form of optimization problem as follows:

$$
\begin{aligned}
& \max _{p=1} \sum_{p \in j^{p}}^{m} y_{j p}^{*} \hat{w}_{p} \omega_{j p} \\
& \text { s.t : }
\end{aligned}
$$

$\sum_{p \in j^{p}} \omega_{j p} \leq \Gamma^{p}$

$0 \leq \omega_{j p} \leq 1 \quad \forall p \in j^{p}$

The dual form of (13) is as follows:

$\min \gamma^{p} \Gamma^{p}+\sum_{p \in j p} \mu_{j p}$

s.t :

$\mu_{j p}+\gamma^{p} \geq y_{j p} \hat{w}_{p} \quad \forall p \in j^{p}$

$\mu_{j p} \geq 0 \quad \forall p \in j^{p}$

$\gamma^{p} \geq 0$

where $\mu_{j p}$ and $\gamma^{p}$ are the dual variables. Considering the obtained linear dual forms of protection functions for uncertain parameter $\tilde{w}_{p}$ in objective function, Constraints 6 and 11 will be modified as follows:

$$
\begin{gathered}
\sum_{i=1}^{n} x_{i j} \leq \frac{T\left(\sum_{p=1}^{m} y_{j p} w_{p}+\gamma^{p} \Gamma^{p}+\sum_{p \in j p} \mu_{j p}\right)}{t_{j}^{\prime}} \quad \forall j \\
\frac{\sum_{i=1}^{n} \sum_{k=1}^{n} f_{i k} \vartheta_{i j k l}}{\left(\sum_{p=1}^{m} y_{j p} w_{p}+\gamma^{p} \Gamma^{p}+\sum_{p \in j p} \mu_{j p}\right)} \leq u \quad \forall j \\
i \neq k, I=j
\end{gathered}
$$


Finally, the proposed robust optimization model is as follows:

$$
\begin{aligned}
& z_{1}=\min \sum_{i=1}^{n} \sum_{\substack{j=1 \\
i \neq k=1}}^{N} \sum_{\substack{l=1 \\
i \neq j \neq l}}^{N} f_{i k} \vartheta_{i j k l} \\
& z_{2}=\min u \\
& z_{3}=\min \sum_{j=1}^{N}\left(\sum_{p=1}^{m} y_{j p} w_{p} \lambda+\gamma^{p} \Gamma^{p}+\sum_{p \in j^{p}} \mu_{j p}\right) \\
& \text { s.t : } \\
& \sum_{j=1}^{N} x_{i j}=1 \quad \forall i
\end{aligned}
$$

$$
\sum_{p=1}^{m} y_{j p}=1 \quad \forall j
$$

$$
\sum_{i=1}^{n} x_{i j} \geq 2 \quad \forall j
$$

$t^{\prime} \geq\left\{f_{i} t_{i} x_{i j}\right\} \quad \forall j$

$$
\sum_{i=1}^{n} x_{i j} \leq \frac{T\left(\sum_{p=1}^{m} y_{j p} w_{p}+\gamma^{p} \Gamma^{p}+\sum_{p \in j p} \mu_{j p}\right)}{t_{j}^{\prime}} \quad \forall j
$$

$x_{i j} \in\{0,1\} \quad \forall j, k$

$y_{j k} \in\{0,1\} \quad \forall j, k$

$$
x_{i j}+x_{j k}-2 * \vartheta_{i j k l} \geq 0 \quad \forall i, j, k, I
$$

$$
\begin{gathered}
\frac{\sum_{i=1}^{n} \sum_{k=1}^{n} f_{i k} \vartheta_{i j k l}}{\left(\sum_{p=1}^{m} y_{j p} w_{p}+\gamma^{p} \Gamma^{p}+\sum_{p \in j p} \mu_{j p}\right)} \leq u \quad \forall j \\
i \neq k, I=j
\end{gathered}
$$

$\mu_{j p}+\gamma^{p} \geq y_{j p} \hat{w}_{p} \quad \forall j, p \in j^{p}$

$\mu_{j p} \geq 0 \quad \forall p \in j^{p}$

$\gamma^{p} \geq 0$

\section{Solution methodology for MLTAGVS}

The proposed mathematical model has a hierarchical objective functions, and solving procedure can be implemented in some steps as follows:

$$
\begin{aligned}
& \operatorname{minf} f_{3} \\
& \text { s.t : } \\
& \left\{\begin{array}{c}
\min f_{2} \\
\text { s.t : } \\
\left\{\begin{array}{c}
f_{1}(y) \leq f_{1}^{\prime *} \\
g_{2}^{i} \leq b_{2}^{i} \\
\operatorname{minf} f_{1} \\
\text { s.t : } \\
g_{1}^{i} \leq b_{1}^{i}
\end{array}\right.
\end{array}\right.
\end{aligned}
$$

In the following approach, initially we minimize interloop transportation by considering part (2a) of objective function and Constraints (2), (3), (4), (7), (8) and (9) which is mentioned as $g_{1}^{i} \leq b_{1}^{i}$; in the next step, we optimize intra-loop transportation, part (2b) of objective function considering related constraints; in the proposed model, all constraint are considered, and plus $f_{1}(y) \leq f_{1}^{\prime *}$. In the last step, result of step 2 can be considered as the optimum assignment of stations to cells, and using the third function, we find the best type of AGV designation to each cell; this methodology is inspired by [24]. The framework of the solution method is illustrated in Fig. 2.

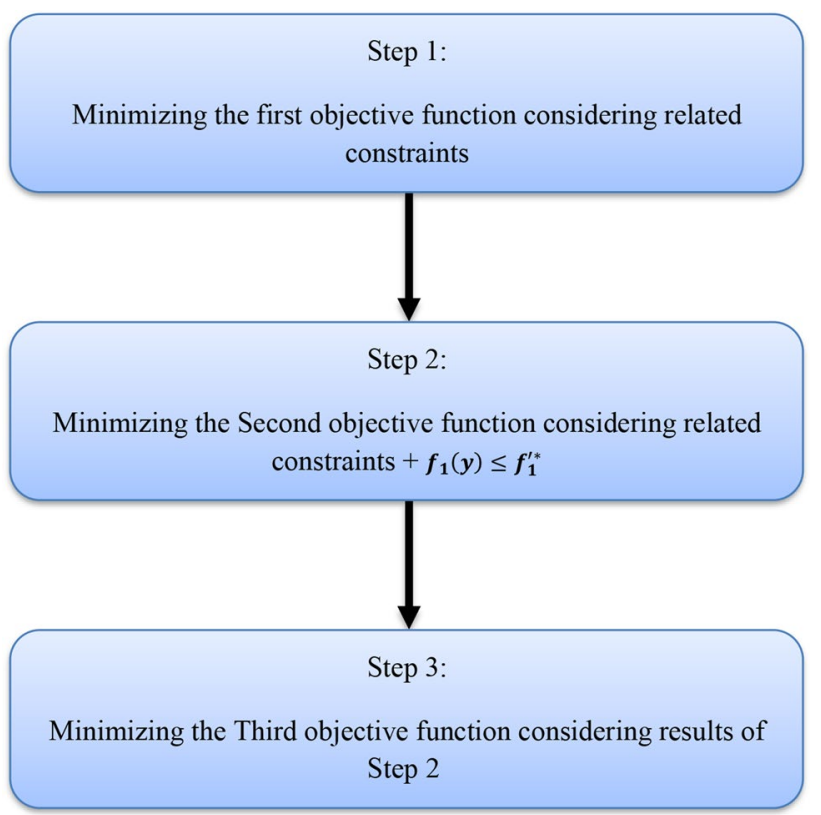

Fig. 2 Framework of solution methodology 
It should be noted that the order of the hierarchy has a great importance and violating this order may create infeasible solution for next step. This methodology is computationally efficient since it considers a subset of constraints in each step, and then, it needs less memory. This method could be an efficient method for bigger problem.

\section{Computational results}

In this section, we apply the solution methodology in Sect. 5 to solve proposed MLTAGVS problem for small-size problem. Note that the solution method is implemented in LINGO software. However, applying meta-heuristic algorithm is out of scope of this study and we are more focused on designing a model to consider uncertainty, but it is good to mention that this problem is a quadratic assignment problem and classified as NP hard combinatorial optimization problem. Thus, applying heuristic and meta-heuristic can improve medium-sized and large-sized problems' solving time significantly.

\subsection{Data generation}

This section devotes to generating necessary data to solve proposed model. The size of problem is determined by number of stations $(I)$, number of cells $(J)$ and number of AGV's $(P)$. In this research, we consider $I=8, J=4$ and $P=3$. The performance coefficients of and AGV and its variation, $\left(w_{p}, \hat{w}_{p}\right)$, for one-to three-load AGV are $(1,0),(1.91,0.06)$, $(2.74,0.17)$ in order, as proposed in [13]. Mean loading, unloading and processing time on station $i\left(t_{i}\right)$ are generated based on a uniform distribution between [10 15]. The penalty cost of $\operatorname{AGV}\left(\lambda_{p}\right)$ is considered as random number in interval [8 12]. The flows from stations are a random number belonging to range [1 10]. The value of $\Gamma^{p}$ is considered equal to 0.5 .

\subsection{Report of the results}

In this section, the results of solving the proposed model applying hierarchical method are presented. This section is divided into three parts: (1) the results of the first step of the solution method, (2) the results of the second step of the solution method and (3) the results of the last step of the solution method.

1. The results of the first step

In this section, the problem is solved only with the first objective function and related constraints. In this state, the
Table 1 Result of assignment of stations to cells

Table 2 Results of the best type of AGV designation to each cell

\begin{tabular}{|c|c|c|c|c|}
\hline \multirow[t]{2}{*}{$Y(j, p)$} & & \multicolumn{2}{|c|}{$\operatorname{AGV}(p)$} & \\
\hline & & 1 & 2 & \\
\hline \multirow[t]{4}{*}{ Cell (j) } & 1 & 0 & 0 & 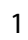 \\
\hline & 2 & 0 & 0 & 1 \\
\hline & 3 & 0 & 1 & 0 \\
\hline & 4 & 0 & 0 & 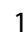 \\
\hline
\end{tabular}

problem is solved in $742 \mathrm{~s}$ and the value of objective function is equal to 245

\section{The results of the second step}

In this part, the problem is solved with the second objective function and all constraints of model are considered and plus $f_{1}(y) \leq f_{1}^{\prime *}$. In this state, the problem is solved in $751 \mathrm{~s}$ and the value of second objective function is equal to 26.28 . The results of assignment of stations to cells are given in Table 1.

\section{The results of the last step}

In this step, the results of step 2 are considered for solving model with the third objective function. Here, we try to find the best capacity to minimize the transportation cost by considering adding AGVs capacity penalty cost. In the final run (last step), the value of the first objective function is equal to 245 , the value of the second objective function is equal to 26.28 and the value of the third objective function is equal to 94.99 . Table 2 shows the results of the best type of AGV designation to each cell.

\subsection{Sensitivity analysis}

In this section, the impact of some parameters $\left(W_{p}, T\right.$ and $\left.f_{i k}\right)$ on the objective functions is investigated. 
For this purpose, a test problem is designed and solved in different situations.

\subsubsection{Sensitivity analysis of the performance coefficient of $\operatorname{AGV}\left(W_{p}\right)$}

We are interested to analyze the impact of AGV performance coefficient, $W_{p}$, on the objective functions. Six different levels of $W_{p}$ are considered, which are base case, $-20 \%,-10 \%,+10 \%$ and $+20 \%$. The sensitivity analysis results are illustrated in Figs. 3, 4 and 5. Figure 3 indicates that the first objective function is not sensitive to $W_{p}$. Since the inter-loop transportations are not affected by AGV's capacity and they will be done by another system, this result could be predicted.

Figure 4 shows the value of the second objective function will decrease as the $W_{p}$ value increases. The intra-loop transportation will be affected by the value of $W_{p}$, because in second objective function, $W_{p}$ is considered as denominator. It is good to mention that this effect significantly depends on penalty cost of increasing AGV capacity in third objective function to assign the optimal capacity in each loop.

According to Fig. 5, increasing in value of $W_{p}$ leads to increase in third objective function value. Since the penalty cost depends on the capacity, by increasing performance, the cost of system will increase.

\subsubsection{Sensitivity analysis of the total available time during work period $(T)$}

This section is devoted to investigate the impact of the total available time during work period on values of objective functions. Table 3 shows the sensitivity analysis of the mentioned parameter.

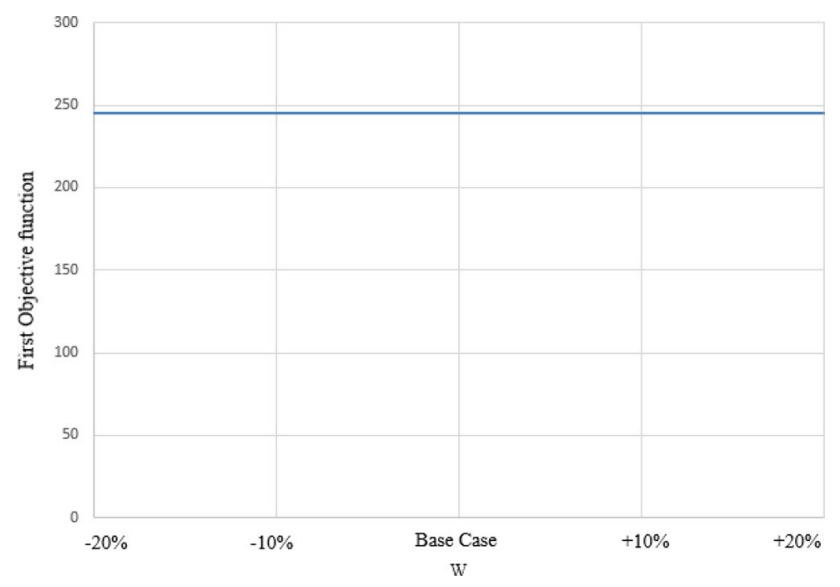

Fig. 3 Sensitivity of the first objective function to the $w_{p}$

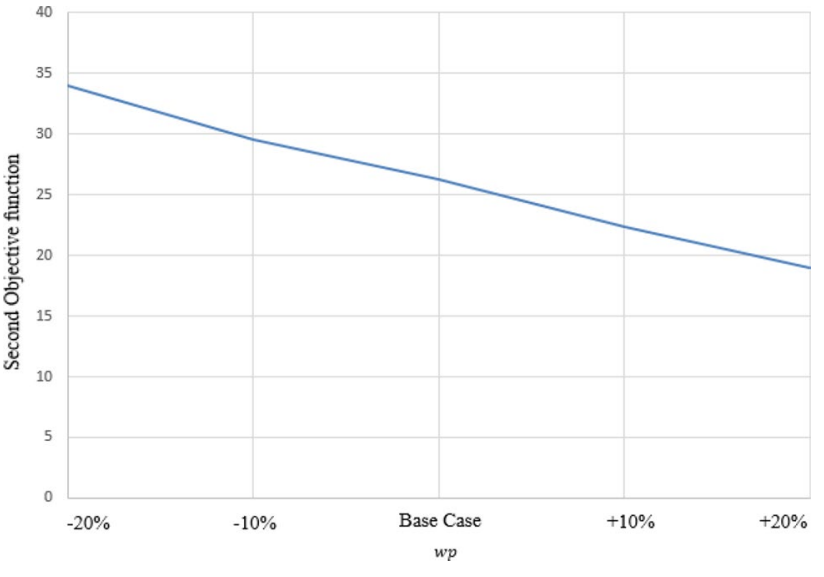

Fig. 4 Sensitivity of the second objective function to the $w_{p}$

As shown in Table 3, changes in the parameter $T$ have no effect on the value of first objective function. On the other hand, the second and third objective function values affected by changing available working time $T$. Basically, by increasing available time either the bottleneck station will change or the transportation in system can be done with the system of AGV with lower capacity, which results in cost reduction. Analyzing the effect of $T$ on the second and third objective function in Table 3 indicates decreasing effect of parameter $T$ on the objective value. By increasing $T$ from base case (500) to 700, the same job can be done in an AGV system with a lower capacity, and consequently, the second and third objective function cutbacks are $8.3 \%$ and $12.51 \%$, respectively. According to Table 3, when the total available working time decreased, the cost initially increased. By decreasing available time, an AGV system with higher capacity is required to perform the transportation job in a limited time interval, so the third objective function cost initially increased. At $T=300$,

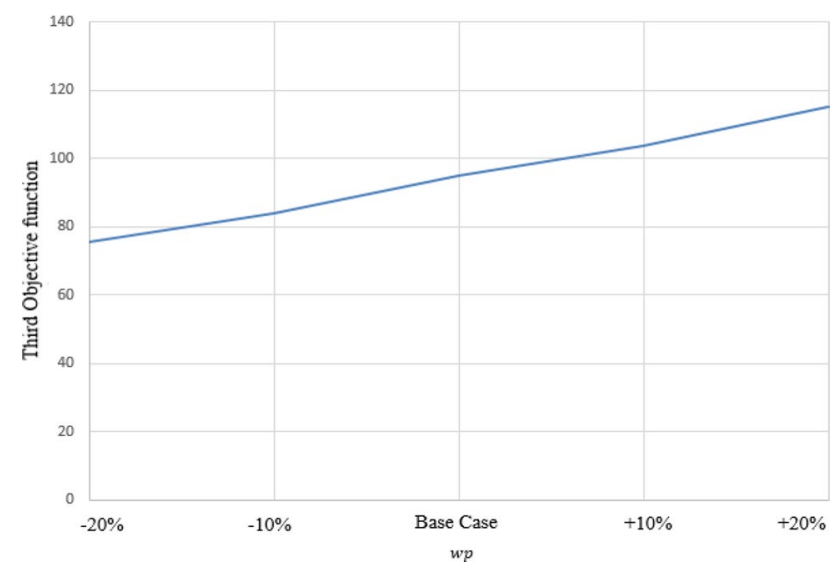

Fig. 5 Sensitivity of the third objective function to the $w_{p}$ 
Table 3 Sensitivity of the objective functions to the parameter $T$

\begin{tabular}{lllllll}
\hline$T$ & OF1 & PCR $^{\text {a }}$ & OF2 & PCR & OF3 & PCR \\
\hline 300 & 245 & $0 \%$ & Infeasible & - & Infeasible & - \\
400 & 245 & $0 \%$ & 27.87 & $6 \%$ & 111.18 & $17.04 \%$ \\
500 (base case) & 245 & - & 26.28 & - & 94.99 & - \\
600 & 245 & $0 \%$ & 24.10 & $-8.3 \%$ & 94.99 & $0 \%$ \\
700 & 245 & $0 \%$ & 24.10 & $-8.3 \%$ & 83.11 & $-12.51 \%$ \\
\hline
\end{tabular}

${ }^{\text {a }}$ Percentage change relative to base case

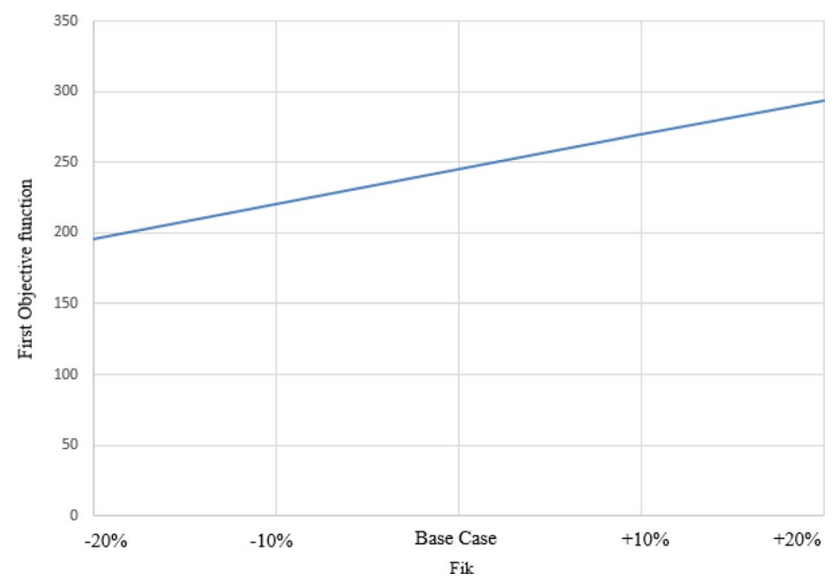

Fig. 6 Sensitivity of the first objective function to the $f_{i k}$

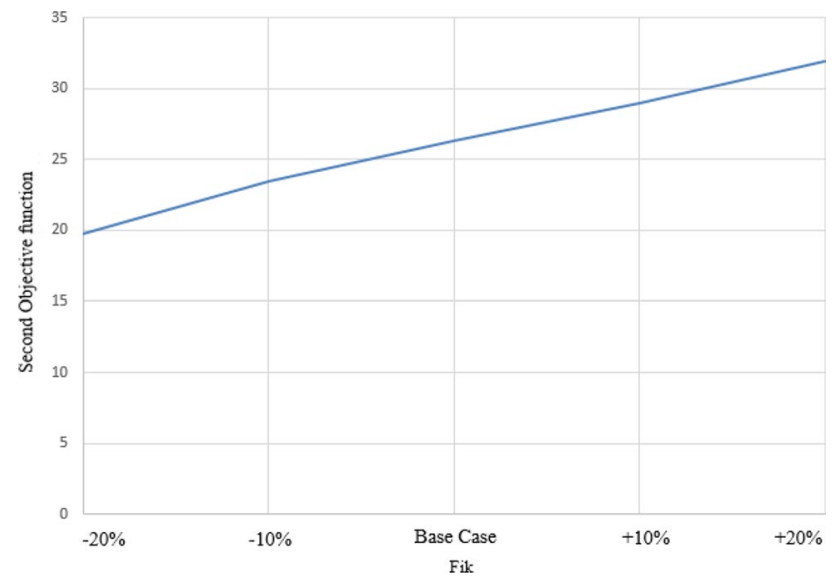

Fig. 7 Sensitivity of the second objective function to the $f_{i k}$

since the available time is less than required time for transportation, the model becomes infeasible.

\subsubsection{Sensitivity analysis of the part flows from station $\left(f_{i k}\right)$}

For analyzing the impact of $f_{i k}$ on the configuration decision, six different levels of $f_{i k}$ are considered, which are base case, $-20 \%,-10 \%,+10 \%$ and $+20 \%$. The sensitivity analysis results are illustrated in Figs. 6, 7 and 8 .

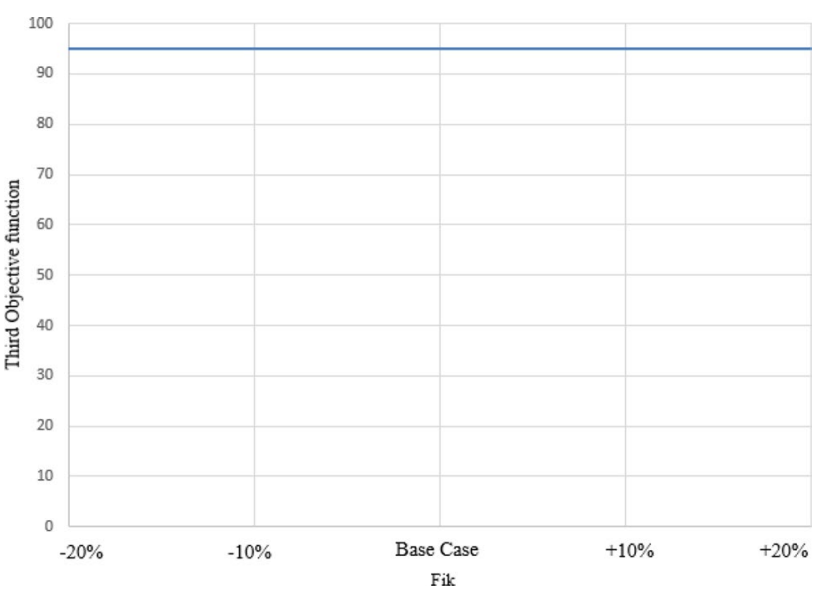

Fig. 8 Sensitivity of the third objective function to the $f_{i k}$

Figures 6 and 7 indicate the increasing effect of part flows on inter-loop and intra-loop transportations, respectively. In this case, since the cost is proportional to the total number of transportation, increase in transportation will grow associated cost.

According to Fig. 8, changes in the $f_{i k}$ in the defined range have no effect in the third objective function, and the assigned AGV system is capable of performing transportation tasks.

\subsubsection{Robustness of solution}

This section presents the validation of robust counterpart and the sensitivity of model to the budget parameter. Figure 9 shows the result of solving problem in nominal state and in robust form when $\Gamma^{p}=0$. As shown in Fig. 9, if the uncertainty budget parameter considered is equal to 0 , the results of nominal and robust models are same.

Also, we investigate the impact of uncertainty budget of objective functions on proposed model.

Figure 10 presents the effect increasing $\Gamma^{p}$ on objective functions values.

Figure 10 shows that the worst objective functions are obtained at the highest value of conservatism degree. The figure indicates that the greater the conservatism degree 


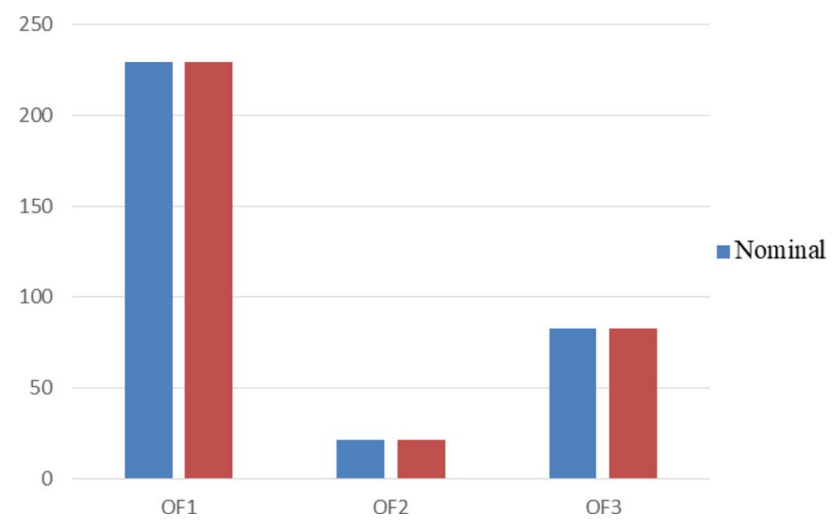

Fig. 9 Comparison objective functions in nominal state and $\Gamma^{p}=0$

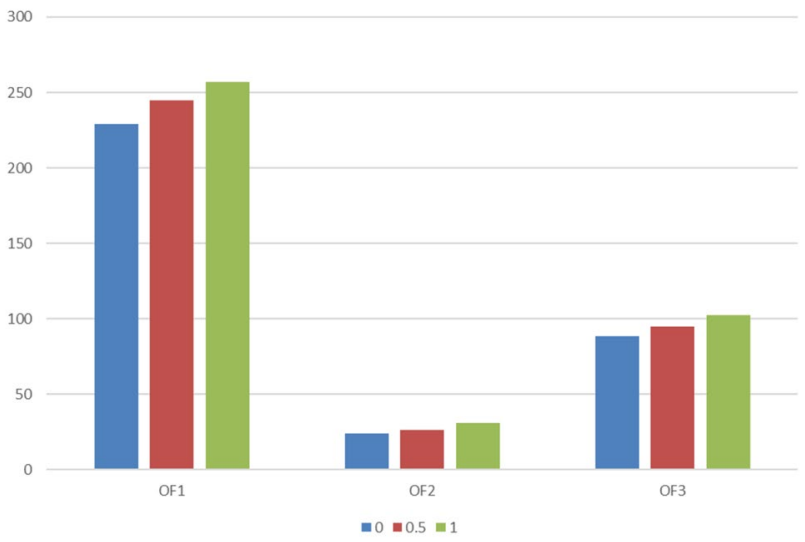

Fig. 10 Sensitivity of model to $\Gamma^{p}$

of performance coefficients is, the larger the impacts will be on objective function values.

\section{Conclusions}

In this paper, a new robust model for designing multiple-load AGV system in tandem configuration has been developed. A comprehensive multi-objective model was proposed to consider all transportation and extending AGV capacity cost. Taking advantage of robust optimization approach, the proposed model was improved to face with variability in AGV performance coefficient. A hierarchical optimization approach is considered to solve the proposed model. The sensitivity analysis of the model indicates that inter-loop transportation cost will not be affected by changing the AGV capacity, but intra-loop transportation will be influenced by this index. In addition, it is shown that increasing uncertainty budget will increase all objective functions. In future research, uncertainty in transportation, availability of AGV due to failure or battery life or uncertainty in production plan could be considered to extend the model. Also, developing evolutionary algorithms is another approach which could be considered for improving solution time for medium- and large-size problems.

\section{Compliance with ethical standards}

Conflict of interest The authors declare that they have no conflict of interest.

\section{References}

1. Asef-Vaziri A, Jahandideh H, Modarres M (2017) Loop-based facility layout design under flexible bay structures. Int J Prod Econ 193:713-725

2. Müller T (1983) Automated guided vehicles. Springer, Berlin

3. Fazlollahtabar H, Saidi-Mehrabad M (2015) Methodologies to optimize automated guided vehicle scheduling and routing problems: a review study. J Intell Robot Syst 77(3-4):525-545

4. Vis IFA (2006) Survey of research in the design and control of automated guided vehicle systems. Eur J Oper Res 170(3):677-709

5. Bozer YA, Srinivasan MM (1989) Tandem configurations for AGV systems offer simplicity and flexibility. Ind Eng 21(2):23-27

6. Salehipour A, Kazemipoor H, Moslemi Naeini L (2011) Locating workstations in tandem automated guided vehicle systems. Int J Adv Manuf Technol 52(1):321-328

7. Shalaby MA, Elmekkawy TY, Fahmy SA (2006) A cost based evaluation of a zones formation algorithm in tandem AGV systems. Int J Adv Manuf Technol 31(1-2):175-187

8. Tavakkoli-Moghaddam MBAR, Kazemipoor H, Salehipour A (2008) Partitioning machines in tandem AGV systems based on 'balanced flow strategy' by simulated annealing. Int J Adv Manuf Technol 38(3-4):355-366

9. Rahimikelarijani B, Saidi-Mehrabad M, Barzinpour F (2018) A mathematical model for multiple-load AGVs in Tandem layout. J Optim Ind Eng 13:67-80

10. Fan X, He Q, Zhang Y (2015) Zone design of Tandem loop AGVs path with hybrid algorithm. IFAC-PapersOnLine 48(3):869-874

11. EIMekkawy TY, Liu S (2009) A new memetic algorithm for optimizing the partitioning problem of tandem AGV systems. Int J Prod Econ 118(2):508-520

12. Salehipour A, Sepehri MM (2014) Optimal location of workstations in tandem automated-guided vehicle systems. Int J Adv Manuf Technol 72(9-12):1429-1438

13. Ho Y-C, Hsieh P-F (2004) A machine-to-loop assignment and layout design methodology for tandem AGV systems with multiple-load vehicles. Int J Prod Res 42(4):801-832

14. Ozden M (1988) A simulation study of multiple-load-carrying automated guided vehicles in a flexible manufacturing system. Int J Prod Res 26(8):1353-1366

15. van der Meer JR (2000) Operational control of internal transport. Erasmus University Rotterdam, Rotterdam

16. Vis IFA (2006) Survey of research in the design and control of automated guided vehicle systems. Eur J Oper Res 170:677-709

17. Fazlollahtabar H, Saidi-mehrabad M, Balakrishnan J (2015) Mathematical optimization for earliness/tardiness minimization in a multiple automated guided vehicle manufacturing system via integrated heuristic algorithms. Robot Auton Syst 72:131-138

18. Afentakis P (1989) A loop layout design problem for flexible manufacturing systems. Int J Flex Manuf Syst 1(2):175-196

\section{SN Applied Sciences}


19. Kim K, Chung B, Jae M (2003) A design for a tandem AGVS with multi-load AGVs. Int J Adv Manuf Technol 22(9):744-752

20. Gabrel V, Murat C, Thiele A (2014) Recent advances in robust optimization: an overview. Eur J Oper Res 235(3):471-483

21. Bertsimas D, Sim M (2004) The price of robustness. Oper Res 52(1):35-53

22. Zokaee S, Jabbarzadeh A, Fahimnia B, Sadjadi SJ (2014) Robust supply chain network design: an optimization model with real world application. Ann Oper Res 257:1-30

23. Soyster AL (1973) Technical note-convex programming with set-inclusive constraints and applications to inexact linear programming. Oper Res 21(5):1154-1157
24. Najafi M, Eshghi K, Dullaert W (2013) A multi-objective robust optimization model for logistics planning in the earthquake response phase. Transp Res Part E 49(1):217-249

Publisher's Note Springer Nature remains neutral with regard to jurisdictional claims in published maps and institutional affiliations. 